\title{
Early Post Operative Septic Arthritis After Hip Arthroscopy Using Tisseel Fibrin Sealant: Report of Two Cases
}

\author{
Patrick Weinrauch $^{\mathrm{a}}$, Sharon Kermecia, ${ }^{\mathrm{a}}$
}

\begin{abstract}
We present two cases of septic arthritis of the hip joint after arthroscopic intervention where both patients were treated with fibrin sealant in association with acetabular micro fracture procedures. Both patients presented with aggressive early onset symptoms, atypical for standard post-operative wound infections. We have now reduced use of Tisseel Fibrin Sealant within the context of hip arthroscopy procedures for concern regarding risk of infection.
\end{abstract}

Keywords: Hip; Arthroscopy; Infection; Septic arthritis; Tisseel

\section{Introduction}

Arthroscopic hip procedures are gaining rapidly in popularity for the management of early symptomatic and prearthritic joint disorders. Symptomatic articular pathology of the hip typically includes damage to the acetabular hyaline cartilage and labrum of the superior-lateral and anterior portions of the joint. In many instances chondral damage may be localised to these specific regions. In situations of focal full thickness chondral loss with otherwise good condition chondral surfaces upon the remainder of the joint, chondral repair or re-growth procedures such as micro-fracture or chondrocyte grafting may be indicated. Chondral grafting procedures using autologous chondrocyte grafting or alternatively acellular collagen matrix scaffolds such as Chondro-Gide for Autologous Matrix Induced Chondrogenesis (AMIC) (LifeHealthcare, North Ryde, NSW, Australia) commonly

\footnotetext{
Manuscript accepted for publication July 18, 2013

a Brisbane Hip Clinic, Level 7, Suite 2, St Andrew's Specialist Centre, St Andrew's War Memorial Hospital, 457 Wickham Terrace, Spring Hill, Queensland, 4000, Australia

${ }^{\mathrm{b}}$ Corresponding author: Sharon Kermeci, Brisbane Hip Clinic, Level

7, Suite 2, St Andrew's Specialist Centre, St Andrew's War

Memorial Hospital, 457 Wickham Terrace, Spring Hill, Queensland, 4000, Australia. Email: skermeci@brisbanehipclinic.com.au
}

doi: http://dx.doi.org/10.4021/jmc1416w requires adhesion to the point of chondral loss by the use of Tisseel fibrin sealant (Baxter AG, Vienna, Austria).

Tisseel sealant has a wide variety of applications in spinal and general surgery for the control of small vessel bleeding by hemostasis. In intra-articular surgery it is commonly used as glue for the adhesion of cellular or non-cellular cartilage repair matrix sheets. Tisseel may also be used for the stabilisation of the autologous fibrin clot associated with the conduct of micro-fracture procedures. Tisseel is delivered by pre-filled single use only syringes within sterile packaging for use during operative procedures. Tisseel syringes are prewarmed in sterile water solution prior to use. Tisseel is administered by cannula directly onto the point of requirement.

Tisseel is produced from pooled human plasma, which in theory may contain infectious agents. The infection risk from human plasma associated with Tisseel use relates predominantly to the potential for viral infection or associated blood pathogens such as Creutzfeldt-Jakob's disease [1]. To our knowledge, bacterial infection and subsequent septic arthritis with articular applications has not been described with the use of Tisseel Fibrin Sealant. We describe the diagnosis of septic arthritis in 2 patients, both of whom were treated with Tisseel Fibrin Sealant as part of their arthroscopic hip procedures for the management of acetabular chondral defects.

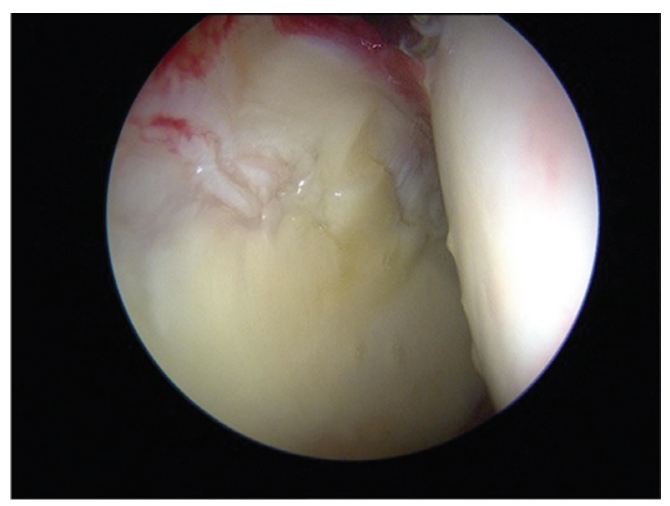

Figure 1. Initial appearance of lesion in case 1. 


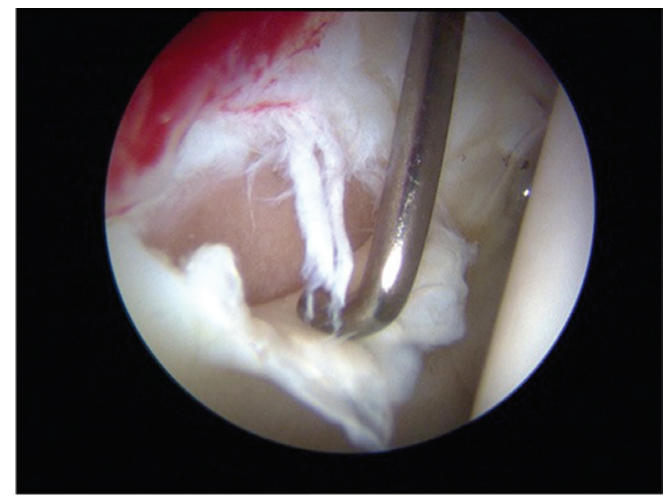

Figure 2. Full thickness delamination of hyline cartilige from acetabular socket.

\section{Case Report}

\section{Case 1}

26-year-old male with cam type femoroacetabular impingement (FAI) of the right hip, presented with symptomatic articular hip pathology consisting of mid-inguinal groin discomfort of a persistent nature which was interfering with his activities and sporting pursuits. On physical examination there was clear evidence of articular pathology of the acetabular rim with a positive provocation Quadrant test and stiffness of the hip range of motion in rotational profile assessment consistent with FAI. CT scans with 2D \& 3D reconstructions demonstrated irregularity of the femoral head neck junction of both hips, correlating with clinically meaningful FAI.

One month following initial evaluation the patient underwent a right hip arthroscopy with arthroscopic femoral osteoplasty. The procedure was undertaken in a supine position with standard two portal central compartment arthroscopic techniques under general anaesthetic. No perioperative antibiotics were administered as per our usual practice during routines arthroscopic hip procedures. Central compartment findings included a region of grade 4 chondral loss from

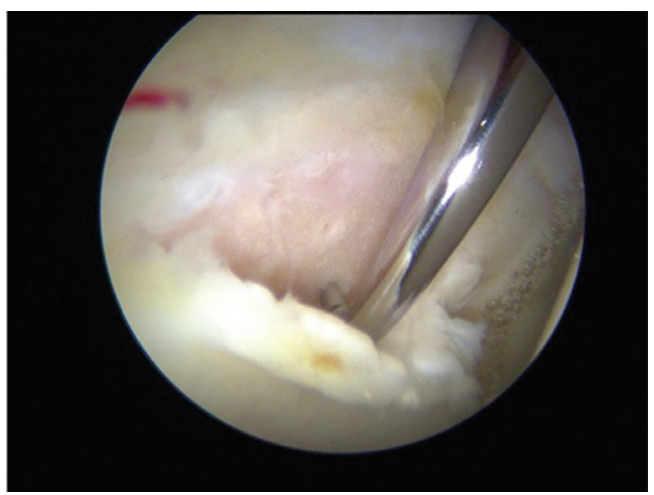

Figure 3. Microfracture behind delaminated chondral flap.

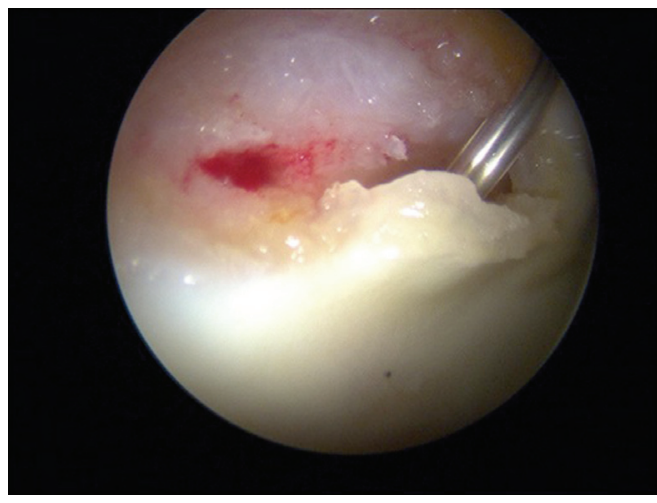

Figure 4. Application of tisseel fibrin sealant behind the chondral flap.

Zone C0 and D0 [2] with a large flap of delaminating full thickness cartilage forming (Fig. 1, 2). In an attempt to retain the large delaminating chondral flap, micro-fracture was conducted behind the lesion and the flap was repaired by Tisseel sealant (Fig. 3, 4, 5). The chondral flap was judged to be stable at the end of repair. Arthroscopic femoral osteoplasty was subsequently conducted via two dedicated peripheral compartment portals after the removal of traction. Total operative time was 75 minutes with 25 minutes under traction. Post procedure, there was a substantial improvement in the internal rotational profile of the hip while in 90 degrees hip flexion from pre-operative 20 degrees to 50 degrees rotation post-osteoplasty.

Postoperatively, the patient was managed in hospital overnight with our standard procedure. Immediate physiotherapy was prescribed for abductor strengthening and peri articular endurance exercises with waterproof dressings reapplied to wounds prior to discharge from hospital.

Within only 36 hours of his operative procedure, the patient represented with increasing severe pain about the hip. Recommendations at this stage were for modification of analgesia however on the basis of progressive deterioration in symptoms, re-admission to hospital at 4 days after the index

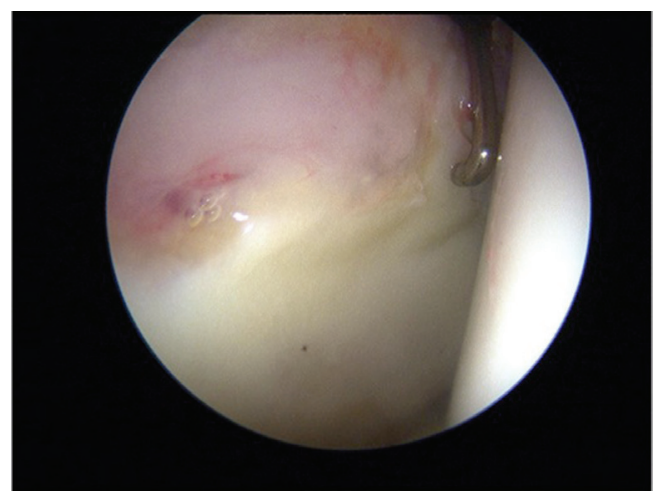

Figure 5. Chondral flap repair with fibrin sealant supporting microfracture clot and cartilage flap. 


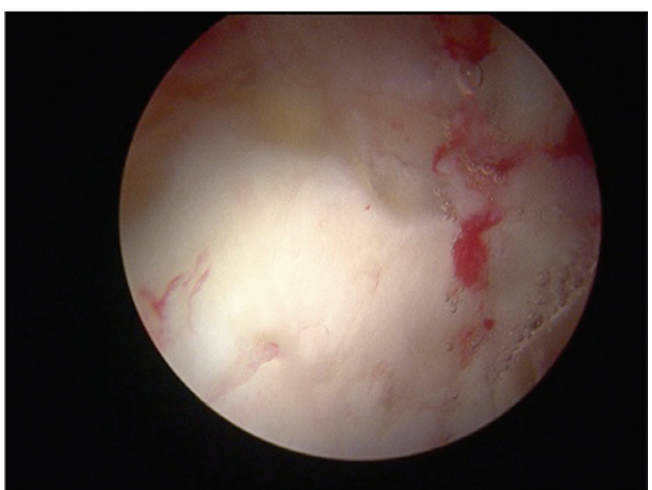

Figure 6. Stable chodral flap at time of resurgery.

procedure was required. At this point, the patient was describing severe pain of an articular nature with clinical signs of deep space infection. White cell count on readmission was $10.2 \times 10^{9} / \mathrm{L}$ (4.0 - 11.0 normal range), C-reactive protein (CRP) $110 \mathrm{mg} / \mathrm{L}$ (0 - 6 normal range) and ESR 67 (1-15 normal range). X-rays and $\mathrm{CT}$ did not demonstrate evidence of fracture. Ultrasound of the hip joint demonstrated an effusion and subsequent culture of aspirate revealed infection with Staphylococcus Caprae. Sensitivity analysis demonstrated the bacteria to be sensitive to all tested antibiotics with the exception of penicillin. Arthroscopic washout of the hip joint was undertaken at 5 days post index procedure and the patient was placed on IV vancomycin. At time of surgery, the chondral repair was judged to be stable (Fig. 6). Patient was changed to IV flucloxacillin in accordance with culture sensitivity and remained in hospital for 7 days post washout. At 17 days post arthroscopic washout for his infection, the patient was faring well. His hip was relatively comfortable throughout mid arc motion and he was comfortably weight bearing with inflammatory markers having normalised. $\mathrm{He}$ was kept on oral antibiotic cover as a precaution for 4 weeks and subsequent to his discontinuing antibiotic therapy, his ESR and CRP remain normal with no recurrence of his infection on clinical grounds. At 3 months post intervention

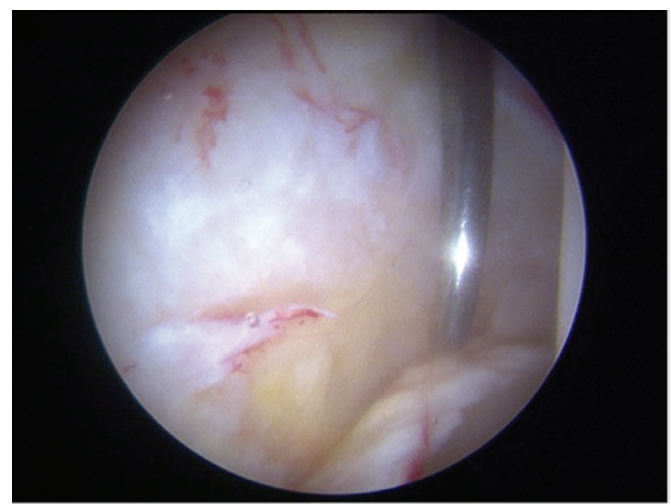

Figure 7. Full thickness chondral flap in case 2.

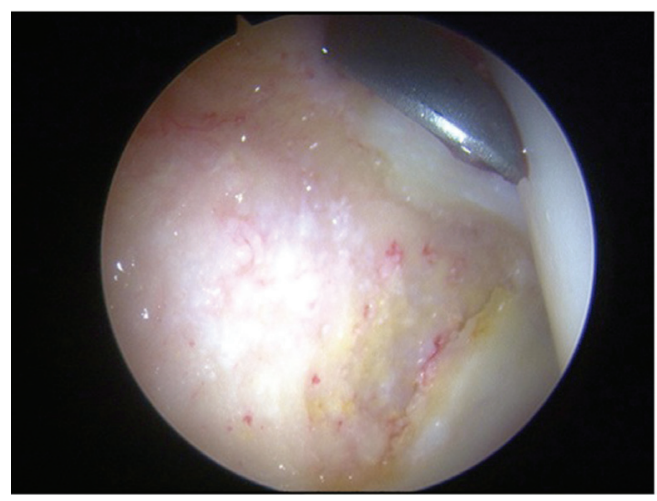

Figure 8. Prepared microfractured surface.

the patient was able to return to high grade physical pursuits including impact sport with relative comfort. There has been no evidence of infection recurrence at beyond 6 months postsurgery.

\section{Case 2}

38-year-old male with bilateral symptomatic FAI, presented with the right side more symptomatic than left. His clinical evaluation included some early joint space narrowing on plain x-ray on the lateral radiograph and MRI scans demonstrated a patch of full thickness hyaline chondral damage from the anterior superior acetabulum in association with an adjacent degenerative acetabular labral tear. The patient had a stiff hip in multiple planes but particularly in rotational profile assessment with an obligatory external rotation contracture in flexion and a positive Quadrant test indicative of damage of the acetabular rim. On the basis of his ongoing symptoms, arthroscopic intervention for his right hip was recommended and conducted 10 weeks after his initial evaluation. The intraoperative central compartment findings demonstrated a grade 4 chondral defect from zones $\mathrm{C} 0$ and D0 upon the acetabular rim, which had also progressed to more medial zones C1 and D1 (Fig. 7). Degenerative acetabular

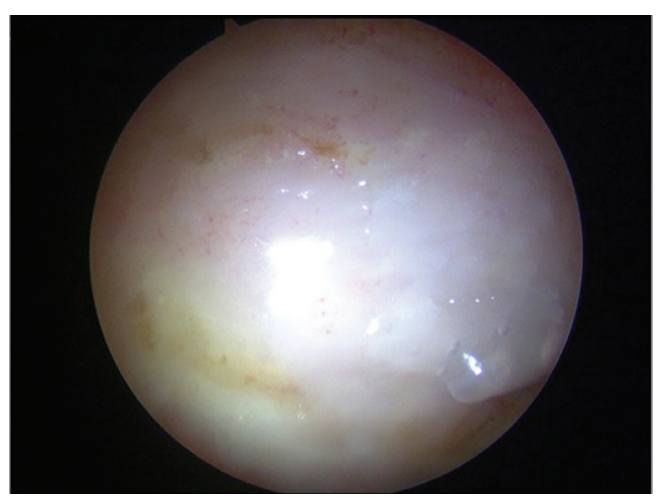

Figure 9. Fibrin sealant in place over microfractured surface. 
labral pathology was debrided. The acetabular hyaline chondral defects were managed by chondroplasty and micro-fracture (Fig. 8). Due to the reasonable size of the micro-fracture area, stabilisation of the micro-fracture clot was conducted by administration of Tisseel onto the micro-fractured surface (Fig. 9). Femoral osteoplasty was felt to be contra-indicated in the context of the more extensive degenerative cartilage damage. On the basis of increasing severe pain and fevers starting at 3 days post surgery, the patient, necessitated readmission to his local hospital. Ultrasound demonstrated no significant effusion within the joint and aspiration on two occasions was unsuccessful. Inflammatory markers at that stage demonstrated a substantial rise in both CRP (186) and also ESR (65) in association with fevers of $38.5^{\circ} \mathrm{C}$. On the basis of his deteriorating clinical picture, fevers and elevated inflammatory markers, the diagnosis of post-operative septic arthritis of the hip joint was made. Intravenous flucloxacillin was started prior to obtaining a tissue or fluid specimen for analysis. The patient was subsequently readmitted to our institution for further management. On admission, repeat ultrasound demonstrated minimal effusion and aspiration of $1 \mathrm{ml}$ of clear fluid was able to be obtained under $\mathrm{x}$-ray guidance. Synovial fluid analysis demonstrated 2+ leucocytes but no bacteria observed on gram stain and subsequent culture revealed no growth (patient was already being treated with intravenous antibiotics). On the basis of only small volumes of fluid observed in serial ultrasound, together with a relatively rapid clinical response to the administration of IV antibiotics, a decision was made to manage his infection by non-operative means. The patient was placed on IV vancomycin for 4 days with relatively rapid clinical improvement and discharged with oral clindamycin $450 \mathrm{mg}$ TDS for a further 4 weeks. All inflammatory markers (ESR, CRP and WCC) were normal at 1 month post surgery. There has been no recurrence of infection at beyond 6 months post-surgery.

\section{Discussion}

Post-operative infections after arthroscopic procedures of the hip are very rare $[3,4]$. Party this is due to the thick soft tissue envelope that surrounds the hip. We have over the last 5 years at our institution conducted greater than 1100 arthroscopic procedures on the hip joint, with no patient experiencing deep space infection until now. We have used Tisseel glue in now only 10 patients of whom 2 have now experienced atypical early onset deep space septic arthritis $(\mathrm{p}<0.001)$. Both of these infections were atypical. They both presented with very early symptoms after intervention, somewhat suggestive that the joints have potentially been inoculated with a dose of bacteria contained within contaminated specimens of Tisseel Fibrin adhesive. While this mechanism of infection cannot be definitively proven, it would explain the early presentation in these patients. An alternative mechanism of action might be that the Tisseel fibrin may act as a scaffold for the adhesion of bacteria allowing infection to subsequently develop. Given our experience, the authors would recommend caution with the use of Tisseel sealants in intra articular surgery, particularly in the presence of other foreign materials such as collagen scaffold matrices.

We would recommend as a precaution that surgeons using Tisseel Fibrin Sealant during arthroscopic joint procedures consider taking culture swabs of the sealant at the time of intervention.

\section{References}

1. Baxter AG. Product information Tisseel (Fibrin Sealant). 2009;1-15; Vienna, Austria.

2. Beck M, Kalhor M, Leunig M, Ganz R. Hip morphology influences the pattern of damage to the acetabular cartilage: femoroacetabular impingement as a cause of early osteoarthritis of the hip. J Bone Joint Surg Br. 2005;87(7):1012-1018.

3. Funke EL, Munzinger U. Complications in hip arthroscopy. Arthroscopy. 1996;12(2):156-159.

4. Griffin DR, Villar RN. Complications of arthroscopy of the hip. J Bone Joint Surg Br. 1999;81(4):604-606. 